\title{
Nuclear astrophysics projects at the low-energy RI beam sepa- rator CRIB
}

H. Yamaguchi ${ }^{1, \star}$, D. Kahl ${ }^{1,2}$, S. Hayakawa ${ }^{1}$, L. Yang ${ }^{1,3}, H$. Shimizu $^{1}$, Y. Sakaguchi ${ }^{1}$, K. Abe ${ }^{1}, T$. Nakao $^{1,4}$, T. Suhara ${ }^{5}$, N. Iwasa ${ }^{6}$, A. Kim ${ }^{7,8}$, D.H. Kim ${ }^{8}$, S.M. Cha7 , M.S. Kwag ${ }^{7}$, J.H. Lee ${ }^{7}$, E.J.

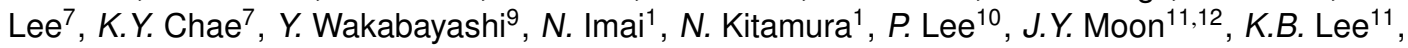
C. Akers ${ }^{11}$, H.S. Jung ${ }^{12}$, N.N. Duy ${ }^{13,14}$, L.H. Khiem ${ }^{13}$, C.S. Lee ${ }^{10}$, T. Hashimoto ${ }^{11}$, S. Kubono ${ }^{9}, T$. Kawabata $^{15}$, T. Teranishi ${ }^{16}$, Y.K. Kwon ${ }^{11}$, and D.N. Binh ${ }^{17,1}$

${ }^{1}$ Center for Nuclear Study (CNS), University of Tokyo, RIKEN campus, 2-1 Hirosawa, Wako, Saitama 3510198, Japan

2 School of Physics and Astronomy, the University of Edinburgh, Peter Guthrie Tait Road, Edinburgh EH9 3BF, UK

${ }^{3}$ China Institute of Atomic Energy, P.O. Box 275(10), Beijing 102413, China

${ }^{4}$ Advanced Science Research Center, Japan Atomic Energy Agency, Tokai, Ibaraki 319-1195, Japan

${ }^{5}$ Matsue College of Technology, Matsue, Shimane 690-8518, Japan

${ }^{6}$ Department of Physics, Tohoku University, Aoba, Sendai, Miyagi 980-8578, Japan

7 Department of Physics, Sungkyunkwan University, 2066 Seobu-ro, Jangan-gu, Suwon, Korea

${ }^{8}$ Department of Physics, Ewha Womans University, Seoul 120-750, Korea

9 The Institute of Physical and Chemical Research (RIKEN), 2-1 Hirosawa, Wako, Saitama 351-0198, Japan

${ }^{10}$ Department of Physics, Chung-Ang University, Seoul 156-756, Korea

${ }^{11}$ Institute for Basic Science, 70, Yuseong-daero 1689-gil, Yuseong-gu, Daejeon 305-811, Korea

12 Wako Nuclear Science Center (WNSC), KEK, 2-1 Hirosawa, Wako, Saitama 351-0198, Japan

${ }^{13}$ Institute of Physics, Vietnam Academy of Science and Technology, 10 Dao Tan, Ba Dinh, Hanoi, Vietnam

${ }^{14}$ Dong Nai University, Le Quy Don Street, Tan Hiep Ward, Bien Hoa City, Dong Nai, Vietnam

${ }^{15}$ Department of Physics, Kyoto University, Kita-Shirakawa, Kyoto 606-8502, Japan

${ }^{16}$ Department of Physics, Kyushu University, 6-10-1 Hakozaki, Fukuoka 812-8581, Japan

1730 MeV Cyclotron Center, Tran Hung Dao Hospital, Hoan Kiem District, Hanoi, Vietnam

\begin{abstract}
Studies on nuclear reactions relevant for astrophysics have been performed using the radioactive-isotope (RI) beams at the low-energy RI beam separator CRIB, operated by Center for Nuclear Study (CNS), the University of Tokyo. A type of measurement to study astrophysical reactions at CRIB is by the elastic resonant scattering with the thick-target method in inverse kinematics. We introduce the $\alpha$ resonant scattering with ${ }^{7} \mathrm{Be}$ beam, related to the astrophysical ${ }^{7} \mathrm{Be}(\alpha, \gamma)$ reactions, which is relevant in the hot $p$ - $p$ chain and $v$-process in supernovae. Other $\alpha$ resonant scattering measurements with ${ }^{30} \mathrm{~S},{ }^{10} \mathrm{Be},{ }^{15} \mathrm{O}$, and ${ }^{18} \mathrm{Ne}$ beams have been performed at CRIB, using the thick-target method.
\end{abstract}

\footnotetext{
^e-mail: yamag@cns.s.u-tokyo.ac.jp
} 


\section{Introduction}

CRIB $[1,2]$ is a radioactive-isotope (RI) beam separator operated by Center for Nuclear Study (CNS), the University of Tokyo, installed at the RIBF facility of RIKEN Nishina Center. CRIB can produce low-energy $(<10 \mathrm{MeV} / \mathrm{u}) \mathrm{RI}$ beams by the in-flight technique, using primary heavy-ion beams accelerated at the AVF cyclotron of RIKEN $(\mathrm{K}=70)$. Most of the RI beams are produced via direct reactions such as $(p, n),(d, p)$ and $\left({ }^{3} \mathrm{He}, n\right)$, taking place at an 8-cm-long gas target with a maximum pressure of 760 Torr. A cryogenic target system, in which the target gas can be cooled down to about $90 \mathrm{~K}$, is currently available, and an intense ${ }^{7} \mathrm{Be}$ beam of $2 \times 10^{8}$ pps was produced using the system [3]. A list of typical parameters of RI beams produced at CRIB is found in [4]. New RI beams recently developed at CRIB are ${ }^{16} \mathrm{~N}\left(1 \times 10^{6} \mathrm{pps}\right),{ }^{10} \mathrm{Be}\left(2 \times 10^{4} \mathrm{pps}\right),{ }^{15} \mathrm{O}\left(1 \times 10^{6} \mathrm{pps}\right)$, and ${ }^{26} \mathrm{Al}\left(1 \times 10^{5}\right.$ pps). The ${ }^{26} \mathrm{Al}$ beam was developed to obtain an isomeric ${ }^{26 m} \mathrm{Al}$ beam, related to the production rate of the galactic $\gamma$ rays from ${ }^{26} \mathrm{Al}$.

A recent major topic of our interest is the measurement of alpha-induced reactions. Several $(\alpha, p)$ reactions, such as ${ }^{14} \mathrm{O}(\alpha, p)[5],{ }^{11} \mathrm{C}(\alpha, p)[6],{ }^{21} \mathrm{Na}(\alpha, p)$, and ${ }^{18} \mathrm{Ne}(\alpha, p)$ have been studied by direct measurement at CRIB. Measurements on the elastic resonant scatterings with a helium target and beams of ${ }^{7} \mathrm{Li}[7]$ and ${ }^{7} \mathrm{Be}[8],{ }^{30} \mathrm{~S},{ }^{10} \mathrm{Be},{ }^{15} \mathrm{O}$, and ${ }^{18} \mathrm{Ne}$ have also been performed. These measurements provide information on the resonances to evaluate astrophysical $(\alpha, \gamma)$ reaction rates, and also on the nuclear cluster structure of the compound nuclei.

\section{$2{ }^{7} \mathrm{Be}+\alpha$ elastic resonant scattering}

Here we introduce the ${ }^{7} \mathrm{Be}+\alpha$ scattering measurement as a typical resonant scattering experiment at CRIB. The measurement allows us to evaluate the rate of the ${ }^{7} \mathrm{Be}(\alpha, \gamma)$ reaction, which is considered to play an important role in the hot $p$ - $p$ chain and related reaction sequences [9]. In the $v p$-process in core-collapse supernovae [10], the ${ }^{7} \operatorname{Be}(\alpha, \gamma)$ reaction may contribute as much as the triple- $\alpha$ process to the synthesis of elements heavier than boron at the relevant temperature of $T_{9}=1.5-3$, according to a theoretical calculation [11]. The Gamow energy window at the highest temperature $T_{9}=3$ corresponds to the excitation energy $E_{\mathrm{ex}}=8.2-9.6 \mathrm{MeV}$ in ${ }^{11} \mathrm{C}$.

We performed the measurement of the ${ }^{7} \mathrm{Be}+\alpha$ resonant elastic and inelastic scatterings with the thick-target method in inverse kinematics at CRIB [8]. A low-energy ${ }^{7} \mathrm{Be}$ beam at $14.7 \mathrm{MeV}$ was produced with a typical intensity of $1-2 \times 10^{5}$ per second. The main measurement was performed for 4 days, injecting $2.9 \times 10^{10}{ }^{7} \mathrm{Be}$ particles into the helium target. We obtained an excitation function of the elastic scattering with several peaks, corresponding to the resonance structure in ${ }^{11} \mathrm{C}$. The obtained excitation is shown in the left panel of Figure 1. An R-matrix analysis was performed to deduce the parameters of the resonances, as the calculated curve also shown in the figure. The resonances observed in the present work might contribute to the astrophysical ${ }^{7} \mathrm{Be}(\alpha, \gamma){ }^{11} \mathrm{C}$ reaction rate at high temperature, $T_{9}>1.5$. The resonant reaction rates were calculated for three resonances using analytical formula described in [12], and plotted in Fig. 1. In conclusion, the resonances at $8.90 \mathrm{MeV}$ and $9.20 \mathrm{MeV}$ have a possibility to give significant contributions to the reaction rate for $T_{9}=1.5-3$, although they are evaluated as one order of magnitude smaller contribution than the dominant $8.420-\mathrm{MeV}$ resonance.

\section{$3{ }^{10} \mathrm{Be}+\alpha$ elastic resonant scattering}

Another study was performed on the ${ }^{10} \mathrm{Be}+\alpha$ system, mainly on the interest of an exotic cluster structure, while ${ }^{10} \mathrm{Be}(\alpha, n)$ or ${ }^{10} \mathrm{Be}(\alpha, \gamma)$ reaction may play a role in the big-bang nucleosynthesis [13] or other high-temperature environments. 

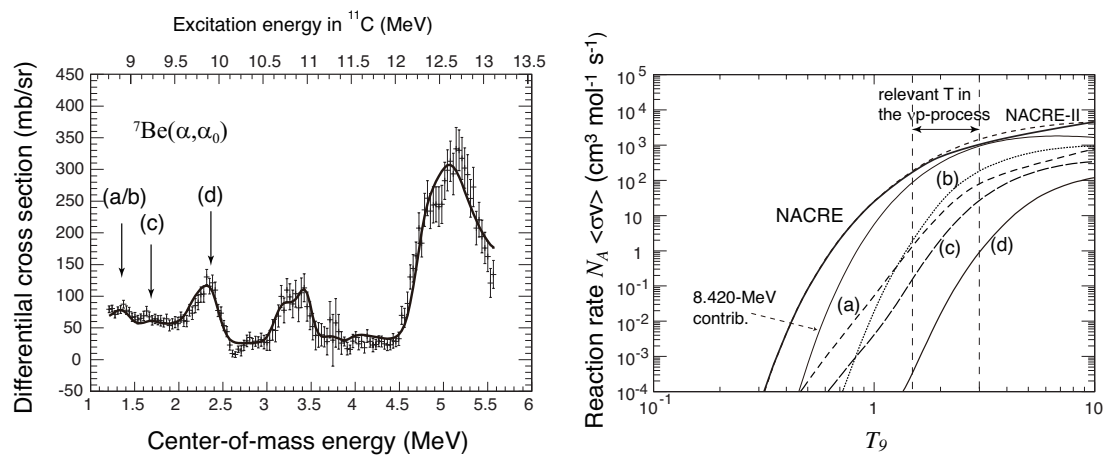

Figure 1. Excitation function of ${ }^{7} \mathrm{Be}+\alpha$ elastic scattering with an R-matrix fit curve (left panel) and resonant reaction rate of ${ }^{7} \mathrm{Be}(\alpha, \gamma)$ for the $8.90,9.20$, and $9.97-\mathrm{MeV}$ resonances, calculated by the analytical formula. The evaluation by NACRE and NACRE-II are shown for comparison. The contribution by the $8.420-\mathrm{MeV}$ resonance, included in NACRE, is also shown.

In 1956, Morinaga [14] came up with the novel idea of a particular cluster state: the linearchain cluster state (LCCS). Now the LCCS is commonly considered as extreme and exotic, due to its presumed propensity to exhibit bending configurations. A theoretical prediction of LCCS in ${ }^{14} \mathrm{C}$ was made by Suhara and En'yo $[15,16]$ with an antisymmetrized molecular dynamics (AMD) calculation, yielding a prolate band $\left(\mathrm{J}^{\pi}=0^{+}, 2^{+}, 4^{+}\right)$that has a configuration of an LCCS at a few MeV or more above the ${ }^{10} \mathrm{Be}+\alpha$ threshold.

We applied the ${ }^{10} \mathrm{Be}+\alpha$ resonant scattering method in inverse kinematics to identify the predicted LCCS band in ${ }^{14} \mathrm{C}$ [17]. The ${ }^{10} \mathrm{Be}$ beam had a typical intensity of $2 \times 10^{4}$ particles per second, and the beam purity was better than $95 \%$. The ${ }^{10} \mathrm{Be}$ beam at $25.8 \mathrm{MeV}$ impinged on the gas target, which was a chamber filled with helium gas at 700 Torr and covered with a $20-\mu \mathrm{m}$-thick Mylar film as the beam entrance window. The measured ${ }^{10} \mathrm{Be}$ beam energy at the entrance of the helium gas target, after the Mylar film, was $24.9 \pm 0.3 \mathrm{MeV}$. $\alpha$ particles recoiling to the forward angles were detected by $\Delta E-E$ detector telescopes. We obtained an excitation function of the ${ }^{10} \mathrm{Be}+\alpha$ resonant elastic scattering for 13.8-19.1 MeV. We performed an R-matrix calculation to deduce the resonance parameters, and we

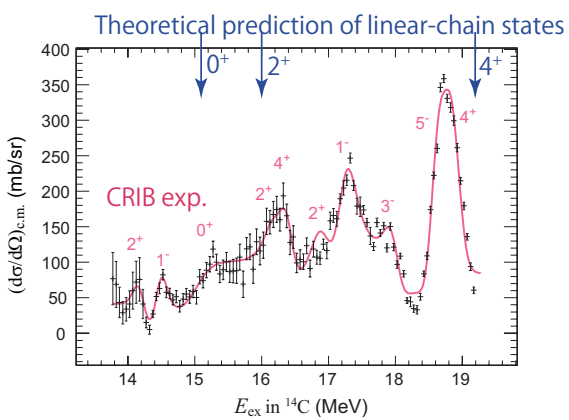

Figure 2. Excitation function of the ${ }^{10} \mathrm{Be}+\alpha$ resonant scattering for $\theta_{\mathrm{lab}}=0-8^{\circ}$.

identified three resonances well-corresponding to the predicted LCCS band; $J^{\pi}$ are identical, and their 
energies and spacings are consistent with the theoretical prediction. The $J^{\pi}$ for the $2^{+}$and $4^{+}$levels were assigned with a complex analysis of unresolved doublets, but they were supported by the angular distribution as well. We claim this as the strongest indication of the LCCS ever found. It can be also shown that both sets of level energies can be plotted almost on a line, $E_{J}=E_{0}+\hbar^{2} / 2 \mathfrak{J}(J(J+1))$, where $\mathfrak{I}$ is the moment of inertia of the nucleus. The linearity allows us to interpret the levels as a rotational band, and the low $\hbar^{2} / 2 \mathfrak{J}=0.19 \mathrm{MeV}$ implies the nucleus could be strongly deformed, consistent with the interpretation of an LCCS.

The experiments were performed at RI Beam Factory operated by RIKEN Nishina Center and CNS, the University of Tokyo. We are grateful to the RIKEN and CNS accelerator staff for their help. This work was partly supported by JSPS KAKENHI (No. 25800125, 15K17662, 16K05369, and 16H03980) from the Ministry of Education, Culture, Sports, Science and Technology (MEXT) of Japan, and the National Research Foundation Grant funded by Korea Government (Grants Nos. NRF-2009-0093817, NRF-2016R1D1A1A09917463, NRF-2016R1A5A1013277, and NRF-2016K1A3A7A09005579).

\section{References}

[1] S. Kubono, Y. Yanagisawa, T. Teranishi, S. Kato, T. Kishida, S. Michimasa, Y. Ohshiro, S. Shimoura, K. Ue, S. Watanabe et al., Eur. Phys. J. A13, 217 (2002)

[2] Y. Yanagisawa, S. Kubono, T. Teranishi, K. Ue, S. Michimasa, M. Notani, J.J. He, Y. Ohshiro, S. Shimoura, S. Watanabe et al., Nucl. Instrum. Meth. Phys. Res., Sect. A 539, 74 (2005)

[3] H. Yamaguchi, Y. Wakabayashi, G. Amadio, S. Hayakawa, H. Fujikawa, S. Kubono, J. He, A. Kim, D. Binh, Nucl. Instrum. Meth. Phys. Res., Sect. A 589, 150 (2008)

[4] D. Kahl, T. Hashimoto, N.N. Duy, S. Kubono, H. Yamaguchi, D.N. Binh, A.A. Chen, S. Cherubini, S. Hayakawa, J.J. He et al., AIP Conference Proceedings 1594, 163 (2014)

[5] A. Kim, N.H. Lee, M.H. Han, J.S. Yoo, K.I. Hahn, H. Yamaguchi, D.N. Binh, T. Hashimoto, S. Hayakawa, D. Kahl et al., Phys. Rev. C 92, 035801 (2015)

[6] S. Hayakawa, S. Kubono, D. Kahl, H. Yamaguchi, D.N. Binh, T. Hashimoto, Y. Wakabayashi, J.J. He, N. Iwasa, S. Kato et al., Phys. Rev. C 93, 065802 (2016)

[7] H. Yamaguchi, T. Hashimoto, S. Hayakawa, D.N. Binh, D. Kahl, S. Kubono, Y. Wakabayashi, T. Kawabata, T. Teranishi, Phys. Rev. C 83, 034306 (2011)

[8] H. Yamaguchi, D. Kahl, Y. Wakabayashi, S. Kubono, T. Hashimoto, S. Hayakawa, T. Kawabata, N. Iwasa, T. Teranishi, Y. Kwon et al., Phys. Rev. C 87, 034303 (2013)

[9] M. Wiescher, J. Görres, S. Graff, L. Buchmann, F.K. Thielemann, Astrophys. J. 343, 352 (1989)

[10] C. Fröhlich, P. Hauser, M. Liebendörfer, G. Martínez-Pinedo, F.K. Thielemann, E. Bravo, N. Zinner, W. Hix, K. Langanke, A. Mezzacappa et al., Astrophys. J. 637, 415 (2006)

[11] S. Wanajo, H.T. Janka, S. Kubono, Astrophys. J. 729, 46 (2011)

[12] C. Angulo et al., Nucl. Phys. A 656, 3 (1999)

[13] A. Coc, S. Goriely, Y. Xu, M. Saimpert, E. Vangioni, Astrophys. J. 744, 158 (2012)

[14] H. Morinaga, Phys. Rev. 101, 254 (1956)

[15] T. Suhara, Y. Kanada-En'yo, Phys. Rev. C 82, 044301 (2010)

[16] T. Suhara, Y. Kanada-En'yo, Phys. Rev. C 84, 024328 (2011)

[17] H. Yamaguchi, D. Kahl, S. Hayakawa, Y. Sakaguchi, K. Abe, T. Nakao, T. Suhara, N. Iwasa, A. Kim, D. Kim et al., Physics Letters B 766, 11 (2017) 